\title{
EXISTENCE OF NONTRIVIAL SOLUTIONS FOR \\ SEMILINEAR PROBLEMS WITH STRICTLY \\ DIFFERENTIABLE NONLINEARITY
}

SERGIO LANCELOTTI

Received 8 March 2005; Accepted 13 July 2005

The existence of a nontrivial solution for semilinear elliptic problems with strictly differentiable nonlinearity is proved. A result of homological linking under nonstandard geometrical assumption is also shown. Techniques of Morse theory are employed.

Copyright (c) 2006 Sergio Lancelotti. This is an open access article distributed under the Creative Commons Attribution License, which permits unrestricted use, distribution, and reproduction in any medium, provided the original work is properly cited.

\section{Introduction}

Since the paper of Amann and Zehnder [1], the existence of nontrivial solutions $u$ for semilinear elliptic problems of the form

$$
-\Delta u=g(u) \quad \text { in } \Omega, \quad u=0 \quad \text { on } \partial \Omega \text {, }
$$

with $g(0)=0$, has been the object of several studies, in which topological and variational methods are successfully applied. We refer the reader to $[2,3,8,10]$. In particular, since the combination of linking theorems and Morse theory has turned out to be very fruitful, it is customary to impose conditions on $g$ that guarantee that the associated functional $f: H_{0}^{1}(\Omega) \rightarrow \mathbb{R}$, given by

$$
f(u)=\frac{1}{2} \int_{\Omega}|D u|^{2} d x-\int_{\Omega} G(u) d x, \quad G(s)=\int_{0}^{s} g(t) d t,
$$

is of class $C^{2}$.

In a recent paper [12], Perera and Schechter have proved a result of Amann-Zehnder type under assumptions that imply $f$ to be only of class $C^{1}$. More precisely, about the regularity of $g$, they assume that $g$ is continuous, there exist in $\mathbb{R}$ the limits

$$
\lim _{s \rightarrow-\infty} \frac{g(s)}{s}, \quad \lim _{s \rightarrow+\infty} \frac{g(s)}{s}, \quad \lim _{s \rightarrow 0} \frac{g(s)}{s}
$$

Hindawi Publishing Corporation

Abstract and Applied Analysis

Volume 2006, Article ID 62458, Pages 1-14

DOI 10.1155/AAA/2006/62458 
2 Existence of nontrivial solutions for semilinear problems

and that

$$
\frac{g(s)}{s} \text { is Lipschitz continuous in a neighbourhood of } 0 \text {. }
$$

One could observe that hypothesis (1.4) allows $f$ not to be of class $C^{2}$, but it does not include every $g$ satisfying the usual assumption that $g$ is of class $C^{1}$ and $g^{\prime}$ is bounded. In particular, condition (1.4) is not stable if we add to $g$ a term of the form

$$
\frac{|s|^{3 / 2}}{1+s^{2}} \text {. }
$$

The first purpose of this paper is to extend the result of [12] in such a way that also the classical smooth case is included. Our result is the following.

TheOREM 1.1. Let $\Omega$ be a bounded open subset of $\mathbb{R}^{n}$ and $g: \mathbb{R} \rightarrow \mathbb{R}$ be a continuous function satisfying $g(0)=0$ and

(a) there exists $C \geq 0$ such that

$$
|g(s)| \leq C(1+|s|)
$$

(b) there exists $\alpha \in \mathbb{R}$ such that

$$
\lim _{s \rightarrow \pm \infty} \frac{g(s)}{s}=\alpha
$$

If we denote by $\left(\lambda_{m}\right)$ the sequence of the eigenvalues of $-\Delta$ with homogeneous Dirichlet boundary condition, let us assume that $\alpha \neq \lambda_{m}$ for any $m \in \mathbb{N}$. Moreover, let us suppose that $g$ is strictly differentiable at 0 (see Definition 3.1 below) and that there exists $m \in \mathbb{N}$ with either $g^{\prime}(0)<\lambda_{m}<\alpha$ or $g^{\prime}(0)>\lambda_{m}>\alpha$.

Then (1.1) admits a nontrivial solution.

Theorem 1.1 is in fact a particular case of a more general result, which will be presented in Section 2.

Remark 1.2. If, as in [12], we have $g(s)=s \gamma(s)$, with $\gamma$ Lipschitz continuous in a neighbourhood of 0 , then it is easy to see that $g$ is strictly differentiable at 0 .

A second purpose of the paper is to improve the saddle theorem proved in [11, Theorem 1.4], also mentioned in [12], in which the functional is of class $C^{2}$, but nonstandard geometrical assumptions are considered. We will prove the following.

Theorem 1.3. Let $H$ be a Hilbert space such that $H=H_{-} \oplus H_{+}$with $\operatorname{dim} H_{-}<\infty$ and $H_{+}$ closed in $H$. Let $f: H \rightarrow \mathbb{R}$ be a functional of class $C^{2}$ and assume that

$$
c_{0}=\inf _{H_{+}} f>-\infty, \quad c_{1}=\sup _{H_{-}} f<+\infty,
$$

$f$ satisfies $(P S)_{c}$ for every $c \in\left[c_{0}, c_{1}\right], f^{\prime \prime}(u)$ is a Fredholm operator at every critical point $u$ in $f^{-1}\left(\left[c_{0}, c_{1}\right]\right)$.

Then there exists a critical point $u$ of $f$ with $c_{0} \leq f(u) \leq c_{1}$ and $m(f, u) \leq \operatorname{dim} H_{-} \leq$ $m^{*}(f, u)$. 
In [11] it is only shown that there exist critical points $\underline{u}$, $\bar{u}$ with $c_{0} \leq f(\bar{u}) \leq f(\underline{u}) \leq$ $c_{1}$ and $m(f, \underline{u}) \leq \operatorname{dim} H_{-} \leq m^{*}(f, \bar{u})$, but one cannot say if there exists a critical point $u=\underline{u}=\bar{u}$, as in the case with standard geometrical assumptions (see [8]), or not. Our improvement is related to the fact that, according to Proposition 4.3 below, also under the nonstandard geometrical assumptions of Theorem 1.3, it is possible to recognize a homological linking structure.

The paper is organized as follows: in Section 2 we state the result of existence of nontrivial solutions; Sections 3 and 4 are devoted to prove some auxiliary results, while in Section 5 we prove the main theorems.

\section{Existence of a nontrivial solution}

Let $\Omega$ be a bounded open subset of $\mathbb{R}^{n}$ and $g: \Omega \times \mathbb{R} \rightarrow \mathbb{R}$ be a Carathéodory function satisfying

$\left(\mathrm{g}_{0}\right) g(x, 0)=0$ for a.e. $x \in \Omega$;

$\left(g_{1}\right)$ there exists $C \geq 0$ such that $|g(x, s)| \leq C(1+|s|)$ for a.e. $x \in \Omega$ and every $s \in \mathbb{R}$;

$\left(\mathrm{g}_{2}\right)$ for a.e. $x \in \Omega$, the function $\{s \mapsto g(x, s)\}$ is strictly differentiable at 0 (see Definition 3.1 below) with $D_{s} g(\cdot, 0) \in L^{\infty}(\Omega)$;

$\left(\mathrm{g}_{3}\right)$ there exist $\widehat{C} \geq 0$ and $\delta>0$ such that, for a.e. $x \in \Omega$, we have

$$
\forall s, t \in]-\delta, \delta[:|g(x, s)-g(x, t)| \leq \hat{C}|s-t|
$$

If we set $G(x, s)=\int_{0}^{s} g(x, t) d t$, it is well known that the functional $f: H_{0}^{1}(\Omega) \rightarrow \mathbb{R}$ defined by

$$
f(u)=\frac{1}{2} \int_{\Omega}|D u|^{2} d x-\int_{\Omega} G(x, u) d x
$$

is of class $C^{1}$.

We denote by $m(f, 0)$ the supremum of the dimensions of the linear subspaces of $H_{0}^{1}(\Omega)$ where the quadratic form

$$
Q(u)=\int_{\Omega}|D u|^{2} d x-\int_{\Omega} D_{s} g(x, 0) u^{2} d x
$$

is negative definite, and by $m^{*}(f, 0)$ the supremum of the dimensions of the linear subspaces of $H_{0}^{1}(\Omega)$ where $Q$ is negative semidefinite. We call $m(f, 0)$ (resp., $m^{*}(f, 0)$ ) the strict (resp., large) Morse index of $f$ at 0 .

Theorem 2.1. Assume that $H_{0}^{1}(\Omega)=X_{-} \oplus X_{+}$with $\operatorname{dim} X_{-}<\infty$ and $X_{+}$closed in $H_{0}^{1}(\Omega)$. Suppose also that

$$
c_{0}=\inf _{X_{+}} f>-\infty, \quad c_{1}=\sup _{X_{-}} f<+\infty,
$$

and that $f$ satisfies $(P S)_{c}$ for every $c \in\left[c_{0}, c_{1}\right]$, 
4 Existence of nontrivial solutions for semilinear problems

If it is $\operatorname{dim} X_{-} \notin\left[m(f, 0), m^{*}(f, 0)\right]$, then the problem

$$
-\Delta u=g(x, u) \quad \text { in } \Omega, \quad u=0 \quad \text { on } \partial \Omega,
$$

admits a nontrivial solution $u$.

Remark 2.2. Under the assumption of Theorem 1.1, it is well known that $f$ satisfies $(P S)_{c}$ for any $c \in \mathbb{R}$ and the geometrical assumptions of Theorem 2.1. Since it is clear that also $\left(\mathrm{g}_{0}\right)-\left(\mathrm{g}_{3}\right)$ are satisfied, Theorem 1.1 is a consequence of Theorem 2.1.

\section{Computations of critical groups}

Definition 3.1. Let $\Phi$ be a map from an open subset $U$ of a normed space $X$ to a normed space $Y$ and let $u \in U$. We say that $\Phi$ is strictly differentiable at $u$ (strongly differentiable in the sense of [6]), if there exists a continuous linear map $L: X \rightarrow Y$ such that

$$
\lim _{\substack{\left(w_{1}, w_{2}\right) \rightarrow(u, u) \\ w_{1} \neq w_{2}}} \frac{\Phi\left(w_{1}\right)-\Phi\left(w_{2}\right)-L\left(w_{1}-w_{2}\right)}{\left\|w_{1}-w_{2}\right\|}=0 .
$$

Of course, in such a case $\Phi$ is Fréchet differentiable at $u$ and $L=\Phi^{\prime}(u)$.

Definition 3.2. Let $\mathbb{K}$ be a field, $X$ be a metric space and $f: X \rightarrow \mathbb{R}$ be a continuous function. For $u \in X$ and $c=f(u)$, let us set

$$
\forall q \in \mathbb{Z}: C_{q}(f, u)=H_{q}\left(f^{c}, f^{c} \backslash\{u\}\right)
$$

where $f^{c}=\{v \in X: f(v) \leq c\}$ and $H_{q}(A, B)$ denotes the $q$ th singular homology group of the pair $(A, B)$, with coefficients in $\mathbb{K}$ (see, e.g., [14]). The vector space $C_{q}(f, u)$ is called the qth critical group of $f$ at $u$. Because of the excision property, we may replace $f$ by $\left.f\right|_{U}$ for any neighborhood $U$ of $u$ in $X$.

Definition 3.3. Let $X$ be a Banach space, $U$ an open subset of $X$ and $f: U \rightarrow \mathbb{R}$ be a function of class $C^{1}$. Let $C$ be a closed subset of $X$ with $C \subseteq U$. We say that $f$ satisfies the Palais-Smale condition ( $(P S)$, for short) on $C$, if every sequence $\left(u_{h}\right)$ in $C$ with $f\left(u_{h}\right)$ bounded and $f^{\prime}\left(u_{h}\right) \rightarrow 0$ admits a convergent subsequence. In the case $C=A=X$, we simply say that $f$ satisfies $(P S)$.

Let $c \in \mathbb{R}$. We say that $f$ satisfies the Palais-Smale condition at level $c\left((P S)_{c}\right.$, for short), if every sequence $\left(u_{h}\right)$ in $U$ with $f\left(u_{h}\right) \rightarrow c$ and $f^{\prime}\left(u_{h}\right) \rightarrow 0$ admits a convergent subsequence.

Let $\Omega$ be a bounded open subset of $\mathbb{R}^{n}(n \geq 3), 1 \leq p<(n+2) /(n-2)$ and $g: \Omega \times \mathbb{R} \rightarrow$ $\mathbb{R}$ be a Carathéodory function satisfying

$\left(\mathrm{g}_{1}^{\prime}\right)$ there exists $C \geq 0$ such that $|g(x, s)| \leq C\left(1+|s|^{p}\right)$ for a.e. $x \in \Omega$ and every $s \in \mathbb{R}$. Let $u_{0} \in H_{0}^{1}(\Omega)$ be an isolated weak solution of the semilinear problem

$$
-\Delta u=g(x, u) \quad \text { in } \Omega, \quad u=0 \quad \text { on } \partial \Omega .
$$


By regularity theory, we automatically have $u_{0} \in L^{\infty}(\Omega)$. Moreover, let us assume that:

$\left(\mathrm{g}_{2}^{\prime}\right)$ for a.e. $x \in \Omega$, the function $\{s \mapsto g(x, s)\}$ is strictly differentiable at $u_{0}(x)$ and $D_{s} g\left(\cdot, u_{0}\right) \in L^{\infty}(\Omega) ;$

$\left(\mathrm{g}_{3}^{\prime}\right)$ there exist $\widehat{C} \geq 0$ and $\delta>0$ such that for a.e. $x \in \Omega$

$$
\forall s, t \in]-\delta, \delta\left[:\left|g\left(x, u_{0}(x)+s\right)-g\left(x, u_{0}(x)+t\right)\right| \leq \hat{C}|s-t| .\right.
$$

Let $f: H_{0}^{1}(\Omega) \rightarrow \mathbb{R}$ be the functional

$$
f(u)=\frac{1}{2} \int_{\Omega}|D u|^{2} d x-\int_{\Omega} G(x, u) d x,
$$

where $G(x, s)=\int_{0}^{s} g(x, t) d t$, and let $Q: H_{0}^{1}(\Omega) \rightarrow \mathbb{R}$ be the quadratic form

$$
Q(u)=\int_{\Omega}|D u|^{2} d x-\int_{\Omega} D_{s} g\left(x, u_{0}\right) u^{2} d x .
$$

Finally, let $m\left(f, u_{0}\right)$ and $m^{*}\left(f, u_{0}\right)$ be defined as in Section 2.

Theorem 3.4. We have that $C_{q}\left(f, u_{0}\right)=\{0\}$ for every $q \leq m\left(f, u_{0}\right)-1$ and every $q \geq$ $m^{*}\left(f, u_{0}\right)+1$.

The proof will be given at the end of the section.

As a first step, we approximate the functional $f$ with suitable functionals $f_{\lambda}$ of class $C^{1}$ with $f_{\lambda}^{\prime}$ strictly differentiable at $u_{0}$ and such that the critical groups of $f_{\lambda}$ at $u_{0}$ are independent of $\lambda$.

Let us denote by $\|\cdot\|_{q}$ the norm of $L^{q}(\Omega)$ and by $\|\cdot\|_{1,2}$ the norm of $H_{0}^{1}(\Omega)$.

Remark 3.5. Up to substitute $g$ with $\tilde{g}: \Omega \times \mathbb{R} \rightarrow \mathbb{R}$ defined by

$$
\tilde{g}(x, s)=g\left(x, u_{0}(x)+s\right)-g\left(x, u_{0}(x)\right),
$$

we may assume that $u_{0}=0$ and that $g(x, 0)=0$.

Lemma 3.6. There exists a constant $\bar{C}>0$ such that, for a.e. $x \in \Omega$ and for any $s \in \mathbb{R}$, we have

$$
|g(x, s)| \leq \bar{C}\left(1+|s|^{p-1}\right)|s| .
$$

Proof. If $0<|s|<\delta$, then by $\left(\mathrm{g}_{3}^{\prime}\right)$ it is

$$
\left|\frac{g(x, s)}{s}\right| \leq \hat{C}
$$

Otherwise, if $|s| \geq \delta$, then it is

$$
\left|\frac{g(x, s)}{s}\right| \leq \frac{C\left(1+|s|^{p}\right)}{|s|} \leq \frac{C}{\delta}+C|s|^{p-1} .
$$

Hence the assertion follows. 
6 Existence of nontrivial solutions for semilinear problems

Now let $\delta>0$ be as in $\left(\mathrm{g}_{3}^{\prime}\right)$ and $\vartheta \in C_{c}^{\infty}(\mathbb{R})$ such that $0 \leq \vartheta \leq 1$, supt $\left.(\vartheta) \subseteq\right]-\delta, \delta[$ and

$$
\begin{gathered}
\vartheta(s)=1 \quad \text { if } s \in\left[-\frac{\delta}{4}, \frac{\delta}{4}\right], \\
0 \leq \vartheta \leq \frac{1}{2} \quad \text { if } s \in[-\delta, \delta] \backslash\left[-\frac{\delta}{2}, \frac{\delta}{2}\right] .
\end{gathered}
$$

For every $\lambda \in[0,1]$ let us define $g_{\lambda}(x, s)=g(x, \vartheta(\lambda s) s)$ and let $f_{\lambda}: H_{0}^{1}(\Omega) \rightarrow \mathbb{R}$ be the functional

$$
f_{\lambda}(u)=\frac{1}{2} \int_{\Omega}|D u|^{2} d x-\int_{\Omega} G_{\lambda}(x, u) d x
$$

where $G_{\lambda}(x, s)=\int_{0}^{s} g_{\lambda}(x, t) d t$. It is clear that:

(a) for every $\lambda>0$ and for a.e. $x \in \Omega$, the function $\left\{s \mapsto g_{\lambda}(x, s)\right\}$ is Lipschitz continuous uniformly with respect to $x$;

(b) for every $\lambda$ and for a.e. $x \in \Omega$, the function $\left\{s \mapsto g_{\lambda}(x, s)\right\}$ is strictly differentiable at 0 with $D_{s} g_{\lambda}(x, 0)=D_{s} g(x, 0)$;

(c) for a.e. $x \in \Omega$, the functions $\left\{(\lambda, s) \mapsto g_{\lambda}(x, s)\right\}$ and $\left\{(\lambda, s) \mapsto G_{\lambda}(x, s)\right\}$ are continuous;

(d) there exists $\bar{C} \geq 0$ such that $\left|g_{\lambda}(x, s)\right| \leq \bar{C}\left(1+|s|^{p}\right),\left|G_{\lambda}(x, s)\right| \leq \bar{C}\left(1+|s|^{p+1}\right)$.

Theorem 3.7. The following facts hold:

(i) for every $\lambda \in[0,1]$, the functional $f_{\lambda}$ is of class $C^{1}$;

(ii) there exists an open bounded neighbourhood $U$ of 0 in $H_{0}^{1}(\Omega)$ such that, for every $\lambda \in[0,1], 0$ is the only critical point of $f_{\lambda}$ in $\bar{U}$;

(iii) for every $\lambda \in] 0,1], f_{\lambda}^{\prime}$ is strictly differentiable at 0 with $\left\langle f_{\lambda}^{\prime \prime}(0) v, v\right\rangle=Q(v)$.

Proof. It is readily seen that assertion (i) holds.

Let us consider assertion (ii). By contradiction, let us assume that there exist $\left(\lambda_{h}\right)$ in $[0,1]$ and $\left(u_{h}\right)$ in $H_{0}^{1}(\Omega)$ with $u_{h} \neq 0$ and $u_{h} \rightarrow 0$ strongly in $H_{0}^{1}(\Omega)$ such that $f_{\lambda_{h}}^{\prime}\left(u_{h}\right)=0$. Up to a subsequence, $\lambda_{h} \rightarrow \lambda$ in $[0,1]$. Since $u_{h}$ is a critical point of $f_{\lambda_{h}}$, we have that $u_{h}$ is a weak solution of

$$
-\Delta u=g_{\lambda_{h}}(x, u) \quad \text { in } \Omega, \quad u=0 \quad \text { on } \partial \Omega .
$$

Let

$$
a_{h}= \begin{cases}\frac{g_{\lambda_{h}}\left(x, u_{h}\right)}{u_{h}} & \text { where } u_{h} \neq 0 \\ 0 & \text { where } u_{h}=0\end{cases}
$$

By Lemma 3.6 it is

$$
\left|a_{h}\right| \leq\left|\frac{g_{\lambda_{h}}\left(x, u_{h}\right)}{u_{h}}\right|=\left|\frac{g\left(x, \vartheta\left(\lambda_{h} u_{h}\right) u_{h}\right)}{u_{h}}\right| \leq \bar{C}\left(1+\left|\vartheta\left(\lambda_{h} u_{h}\right) u_{h}\right|^{p-1}\right) \leq \bar{C}\left(1+\left|u_{h}\right|^{p-1}\right) .
$$


Since $u_{h}$ is bounded in $L^{2 n /(n-2)}(\Omega)$, then $a_{h}$ belongs to $L^{q}(\Omega)$ with $q>n / 2$ and

$$
\left\|a_{h}\right\|_{q} \leq C^{\prime}\left(1+\left\|u_{h}\right\|_{2 n /(n-2)}^{p-1}\right) \leq M
$$

Hence $u_{h}$ is a weak solution of the linear problem

$$
-\Delta u=a_{h} u \quad \text { in } \Omega, \quad u=0 \quad \text { on } \partial \Omega .
$$

By [7, Theorem 3.13.1] $u_{h} \in L^{\infty}(\Omega)$ and there exists $C>0$ such that $\left\|u_{h}\right\|_{\infty} \leq C\left\|D u_{h}\right\|_{2}$. Hence $u_{h} \rightarrow 0$ in $L^{\infty}(\Omega)$. Since $\vartheta=1$ on $[-\delta / 4, \delta / 4]$, for $h$ sufficiently large we have that $u_{h}$ is a weak solution of (3.3). It follows that 0 is not an isolated solution of (3.3): a contradiction.

Finally, let us consider assertion (iii). Let $L: H_{0}^{1}(\Omega) \rightarrow H^{-1}(\Omega)$ be the continuous linear operator such that

$$
\langle L v, w\rangle=\langle L w, v\rangle, \quad\langle L v, v\rangle=Q(v)
$$

Let $\left(u_{h}\right),\left(v_{h}\right),\left(w_{h}\right)$ in $H_{0}^{1}(\Omega)$ be such that $u_{h} \rightarrow 0, w_{h} \rightarrow 0$ in $H_{0}^{1}(\Omega)$ and $\left\|v_{h}\right\|_{1,2} \leq 1$. Up to a subsequence, $w_{h} \rightarrow 0$ and $u_{h} \rightarrow 0$ a.e. in $\Omega$. We have that

$$
\begin{aligned}
& \left|\left\langle f_{\lambda}^{\prime}\left(w_{h}\right), v_{h}\right\rangle-\left\langle f_{\lambda}^{\prime}\left(u_{h}\right), v_{h}\right\rangle-\left\langle L\left(w_{h}-u_{h}\right), v_{h}\right\rangle\right| \\
& =\left|\int_{\left\{x \in \Omega: w_{h}(x) \neq u_{h}(x)\right\}}\left[\frac{g_{\lambda}\left(x, w_{h}\right)-g_{\lambda}\left(x, u_{h}\right)}{w_{h}-u_{h}}-D_{s} g(x, 0)\right]\left(w_{h}-u_{h}\right) v_{h} d x\right| \\
& \leq \\
& \quad C\left(\int_{\left\{x \in \Omega: w_{h}(x) \neq u_{h}(x)\right\}}\left|\frac{g_{\lambda}\left(x, w_{h}\right)-g_{\lambda}\left(x, u_{h}\right)}{w_{h}-u_{h}}-D_{s} g(x, 0)\right|^{n / 2} d x\right)^{2 / n} \\
& \quad \times\left\|w_{h}-u_{h}\right\|_{1,2}\left\|v_{h}\right\|_{1,2} .
\end{aligned}
$$

Then it is

$$
\begin{aligned}
& \frac{\left|\left\langle f_{\lambda}^{\prime}\left(w_{h}\right), v_{h}\right\rangle-\left\langle f_{\lambda}^{\prime}\left(u_{h}\right), v_{h}\right\rangle-\left\langle L\left(w_{h}-u_{h}\right), v_{h}\right\rangle\right|}{\| w_{h}-u_{h}||_{1,2}} \\
& \quad \leq C\left(\int_{\left\{x \in \Omega: w_{h}(x) \neq u_{h}(x)\right\}}\left|\frac{g_{\lambda}\left(x, w_{h}\right)-g_{\lambda}\left(x, u_{h}\right)}{w_{h}-u_{h}}-D_{s} g(x, 0)\right|^{n / 2} d x\right)^{2 / n}\left\|v_{h}\right\|_{1,2} \\
& \quad \leq C\left(\int_{\Omega}\left|\frac{g_{\lambda}\left(x, w_{h}\right)-g_{\lambda}\left(x, u_{h}\right)}{w_{h}-u_{h}}-D_{s} g(x, 0)\right|^{n / 2} \chi_{\left\{x \in \Omega: w_{h}(x) \neq u_{h}(x)\right\}} d x\right)^{2 / n} .
\end{aligned}
$$


8 Existence of nontrivial solutions for semilinear problems

By (a) and (b) we can apply Lebesgue's theorem, obtaining

$$
\left(\int_{\Omega}\left|\frac{g_{\lambda}\left(x, w_{h}\right)-g_{\lambda}\left(x, u_{h}\right)}{w_{h}-u_{h}}-D_{s} g(x, 0)\right|^{n / 2} \chi_{\left\{x \in \Omega: w_{h}(x) \neq u_{h}(x)\right\}} d x\right)^{2 / n} \longrightarrow 0
$$

Therefore

$$
\lim _{h \rightarrow+\infty} \frac{\left\langle f_{\lambda}^{\prime}\left(w_{h}\right), v_{h}\right\rangle-\left\langle f_{\lambda}^{\prime}\left(u_{h}\right), v_{h}\right\rangle-\left\langle L\left(w_{h}-u_{h}\right), v_{h}\right\rangle}{\left\|w_{h}-u_{h}\right\|_{1,2}}=0
$$

and assertion (iii) follows.

Theorem 3.8. The critical groups $C_{q}\left(f_{\lambda}, 0\right)$ are independent of $\lambda$. In particular

$$
\forall q \in \mathbb{Z}: C_{q}(f, 0) \approx C_{q}\left(f_{1}, 0\right)
$$

Proof. Let $U$ be an open bounded neighbourhood of 0 in $H_{0}^{1}(\Omega)$ as in assertion (ii) of Theorem 3.7. We claim that if $\lambda_{h} \rightarrow \lambda$ in $[0,1]$, then $\left\|f_{\lambda_{h} \mid \bar{U}}-f_{\lambda \mid \bar{U}}\right\|_{1, \infty} \rightarrow 0$. Let $\left(u_{h}\right)$ be a sequence in $\bar{U}$. Up to a subsequence, $u_{h} \rightarrow u$ in $H_{0}^{1}(\Omega)$ and $u_{h} \rightarrow u$ a.e in $\Omega$. It is

$$
\begin{aligned}
f_{\lambda_{h}}\left(u_{h}\right)-f_{\lambda}\left(u_{h}\right) & =\int_{\Omega}\left[G_{\lambda_{h}}\left(x, u_{h}\right)-G_{\lambda}\left(x, u_{h}\right)\right] d x \\
& =\int_{\Omega}\left[G_{\lambda_{h}}\left(x, u_{h}\right)-G_{\lambda}(x, u)\right] d x+\int_{\Omega}\left[G_{\lambda}(x, u)-G_{\lambda}\left(x, u_{h}\right)\right] d x
\end{aligned}
$$

By (c), (d) and Lebesgue's theorem we deduce that

$$
\int_{\Omega}\left[G_{\lambda_{h}}\left(x, u_{h}\right)-G_{\lambda}(x, u)\right] d x \longrightarrow 0
$$

Therefore $f_{\lambda_{h}} \rightarrow f_{\lambda}$ uniformly on $\bar{U}$.

Now, let $v_{h} \in H_{0}^{1}(\Omega)$ with $\left\|v_{h}\right\|_{1,2} \leq 1$. Up to a subsequence $v_{h} \rightarrow v$ in $H_{0}^{1}(\Omega), v_{h} \rightarrow v$ in $L^{2 n /(n-2)}(\Omega)$ and $v_{h} \rightarrow v$ a.e. in $\Omega$. It is

$$
\begin{aligned}
& \left|\left\langle f_{\lambda_{h}}^{\prime}\left(u_{h}\right), v_{h}\right\rangle-\left\langle f_{\lambda}^{\prime}\left(u_{h}\right), v_{h}\right\rangle\right| \\
& \quad=\left|\int_{\Omega}\left[g_{\lambda_{h}}\left(x, u_{h}\right)-g_{\lambda}\left(x, u_{h}\right)\right] v_{h} d x\right| \\
& \quad=\left|\int_{\Omega}\left[g\left(x, \vartheta\left(\lambda_{h} u_{h}\right) u_{h}\right)-g\left(x, \vartheta\left(\lambda u_{h}\right) u_{h}\right)\right] v_{h} d x\right| \\
& \quad \leq C\left(\int_{\Omega}\left|g\left(x, \vartheta\left(\lambda_{h} u_{h}\right) u_{h}\right)-g\left(x, \vartheta\left(\lambda u_{h}\right) u_{h}\right)\right|^{2 n /(n+2)} d x\right)^{(n+2) / 2 n}\left\|v_{h}\right\|_{1,2} .
\end{aligned}
$$


As before we have that

$$
\int_{\Omega}\left|g_{\lambda_{h}}\left(x, u_{h}\right)-g_{\lambda}\left(x, u_{h}\right)\right|^{2 n /(n+2)} d x \longrightarrow 0 .
$$

It follows that $f_{\lambda_{h}}^{\prime} \rightarrow f_{\lambda}^{\prime}$ uniformly on $\bar{U}$. Finally, since $U$ is bounded and $g$ has subcritical growth, we have that for every $\lambda \in[0,1] f_{\lambda}$ satisfies $(P S)$ in $\bar{U}$. By [5, Theorem 5.2] the assertion follows.

In the second part of this section we deduce from [6] a generalization of the classical Shifting theorem (see [3, Theorem I.5.4], [10, Theorem 8.4]).

Let $H$ be a Hilbert space, $U$ be an open subset of $H, u_{0} \in U$ and $f: U \rightarrow \mathbb{R}$ be a function of class $C^{1}$ such that $f^{\prime}$ is strictly differentiable at $u_{0}$ and $f^{\prime \prime}\left(u_{0}\right)$ is a Fredholm operator. In particular, $f^{\prime}$ is Lipschitz continuous in a neighbourhood of $u_{0}$. Let $L: H \rightarrow H$ be the linear operator defined by

$$
\forall v, w \in H:\langle L v, w\rangle=\left\langle f^{\prime \prime}\left(u_{0}\right) v, w\right\rangle
$$

let $V_{0}=\operatorname{ker} L$ and let $P_{V_{0}}$ be the orthogonal projection on $V_{0}$. We also denote by $m\left(f, u_{0}\right)$ (resp., $\left.m^{*}\left(f, u_{0}\right)\right)$ the strict (resp., large) Morse index of $f$ at $u_{0}$.

THEOREM 3.9. Let $u_{0}$ be an isolated critical point of $f$. Then there exist a neighbourhood $\hat{U}$ of $P_{V_{0}} u_{0}$ in $V_{0}$ and a function $\hat{f}: \hat{U} \rightarrow \mathbb{R}$ of class $C^{1}$ with locally Lipschitz gradient such that $P_{V_{0}} u_{0}$ is an isolated critical point of $\hat{f}$ and

$$
\begin{gathered}
\forall q \in \mathbb{Z}: C_{q}\left(f, u_{0}\right) \approx \begin{cases}C_{q-m\left(f, u_{0}\right)}\left(\hat{f}, P_{V_{0}} u_{0}\right) & \text { if } m\left(f, u_{0}\right)<\infty, \\
\{0\} & \text { if } m\left(f, u_{0}\right)=\infty,\end{cases} \\
\forall q \leq m\left(f, u_{0}\right)-1: C_{q}\left(f, u_{0}\right)=\{0\}, \\
\forall q \geq m^{*}\left(f, u_{0}\right)+1: C_{q}\left(f, u_{0}\right)=\{0\} .
\end{gathered}
$$

Proof. Without loss of generality, we may assume that $u_{0}=0$. From [6, Theorem 1.2] we also see that the generalized Morse lemma holds also in this setting. Arguing as in the proof of [10, Theorem 8.4], we find that (3.29) holds. Actually, in our case $f$ is of class $C^{2-0}$ instead of $C^{2}$, but the proof of [10, Theorem 8.4] remains valid also in this case.

On the other hand, also the proof of [10, Theorem 8.5] can be easily adapted from the $C^{2}$ to the $C^{2-0}$ case. Therefore we have that $C_{q}\left(\hat{f}, P_{V_{0}} u_{0}\right)=\{0\}$ if $q \geq \operatorname{dim} V_{0}+1$. Since $m^{*}\left(f, u_{0}\right)=m\left(f, u_{0}\right)+\operatorname{dim} V_{0}$, the other assertions follow from (3.29).

Finally, let us prove Theorem 3.4.

Proof. By Remark 3.5 we may assume that $u_{0}=0$. Let $f_{\lambda}: H_{0}^{1}(\Omega) \rightarrow \mathbb{R}$ be as in (3.12). By Theorem 3.7 we have that $f_{1}$ is of class $C^{1}$ with $f_{1}^{\prime}$ strictly differentiable at 0 and 0 is an isolated critical point of $f_{1}$. Moreover, $f_{1}^{\prime \prime}(0)$ is a Fredholm operator. By Theorem 3.8 it is

$$
\forall q \in \mathbb{Z}: C_{q}(f, 0) \approx C_{q}\left(f_{1}, 0\right)
$$


On the other hand, since $Q(u)=\left\langle f_{1}^{\prime \prime}(0) u, u\right\rangle$, we have that $m(f, 0)=m\left(f_{1}, 0\right)$ and $m^{*}(f$, $0)=m^{*}\left(f_{1}, 0\right)$. From Theorem 3.9 the assertion follows.

\section{Homological linking}

Throughout this section, $X$ will denote a Banach space, $\mathrm{B}_{r}(u)$ the open ball of center $u \in X$ and radius $r$ and $f: X \rightarrow \mathbb{R}$ a function of class $C^{1}$. We set $K=\left\{u \in X: f^{\prime}(u)=0\right\}$ and, for every $c \in \mathbb{R}$,

$$
K_{c}=\left\{u \in X: f^{\prime}(u)=0, f(u)=c\right\} .
$$

We also denote by $H_{*}$ singular homology.

First of all, let us recall from [4] an extension of the homological linking of [3].

Definition 4.1. Let $D, S, A$ be three subsets of $X, m \in \mathbb{N}$ and $\mathbb{K}$ a field. We say that $(D, S)$ links $A$ homologically in dimension $m$ (over $\mathbb{K}$ ), if $S \subseteq D, S \cap A=\varnothing$ and there exists $z \in H_{m}(X, S ; \mathbb{K})$ belonging to the image of $i_{*}: H_{m}(D, S ; \mathbb{K}) \rightarrow H_{m}(X, S ; \mathbb{K})$ but not of $j_{*}: H_{m}(X \backslash A, S ; \mathbb{K}) \rightarrow H_{m}(X, S ; \mathbb{K})$, where $i:(D, S) \rightarrow(X, S)$ and $j:(X \backslash A, S) \rightarrow(X, S)$ are the inclusion maps.

It is clear that, if $(D, S)$ links $A$ homologically, then $D \cap A \neq \varnothing$.

Theorem 4.2. Let $D, S, A$ be three subsets of $X$ such that $(D, S)$ links $A$ homologically in dimension $m$ and let $z \in H_{m}(X, S ; \mathbb{K})$ be as in Definition 4.1. Assume that

$$
\inf _{A} f>-\infty, \quad \sup _{D} f<+\infty, \quad \forall u \in S: f(u)<\inf _{A} f
$$

and define

$$
\begin{aligned}
& c=\inf \left\{b \in \mathbb{R}: S \subseteq f^{b} \text { and } z\right. \text { belongs to the image of the } \\
& \left.\qquad \text { homomorphism induced by inclusion } H_{m}\left(f^{b}, S ; \mathbb{K}\right) \longrightarrow H_{m}(X, S ; \mathbb{K})\right\} .
\end{aligned}
$$

Suppose that $f$ satisfies (PS) and that each element of $K_{c}$ is isolated in $K$.

Then $\inf _{A} f \leq c \leq \sup _{D} f$ and there exists $u \in K_{c}$ with $C_{m}(f, u) \neq\{0\}$.

To prove our main results we need the following.

Proposition 4.3. Let $X=X_{-} \oplus X_{+}$, with $\operatorname{dim} X_{-}<\infty$ and $X_{+}$closed in $X$. Assume that

$$
c_{0}=\inf _{X_{+}} f>-\infty, \quad c_{1}=\sup _{X_{-}} f<+\infty
$$

and that $f$ satisfies $(P S)_{c}$ for every $c \in\left[c_{0}, c_{1}\right]$.

Then there exists a compact pair $(D, S)$ in $X$ such that

$$
\max _{D} f \leq c_{1}, \quad \forall u \in S: f(u)<c_{0}
$$

and such that $(D, S)$ links $X_{+}$homologically in dimension $\operatorname{dim} X_{-}$over all $\mathbb{K}$. 
Proof. Since $f$ satisfies $(P S)_{c}$ for every $c \in\left[c_{0}, c_{1}\right]$, there exists $r>0$ such that $K \cap f^{-1}\left(\left[c_{0}\right.\right.$, $\left.\left.c_{1}\right]\right) \subseteq\left(\mathrm{B}_{r}(0) \cap X_{-}\right) \oplus X_{+}$. Moreover, there exist $\delta, \sigma>0$ such that

$$
\begin{gathered}
\left\|P_{X_{-}} u\right\| \geq r, \\
c_{0}-\delta \leq f(u) \leq c_{1}+\delta
\end{gathered} \Longrightarrow\left\|f^{\prime}(u)\right\|>\sigma,
$$

where $P_{X_{-}}$denotes the projection on $X_{-}$induced by the decomposition $X=X_{-} \oplus X_{+}$. Let $c>0$ be such that $\left\|P_{X_{-}} u\right\| \leq c\|u\|$ for any $u \in X$ and let

$$
\begin{gathered}
R=c \frac{c_{1}-c_{0}+\delta}{\sigma}+r+\delta, \quad \rho_{1}=1, \quad \rho_{2}=R-r-\delta, \\
C=X \backslash\left[\left(\mathrm{B}_{r+\rho_{1}+\rho_{2}}(0) \cap X_{-}\right) \oplus X_{+}\right] .
\end{gathered}
$$

By [5, Theorem 2.1] applied to the function $f_{\mid\left\{u \in X: f(u) \geq c_{0}-\delta\right\}}$, there exist a continuous function

$$
\tau: \overline{\mathrm{B}_{\rho_{1}}(C)} \cap\left\{u \in X: c_{0}-\delta \leq f(u)<c_{1}+\delta\right\} \longrightarrow[0,+\infty)
$$

and a continuous map

$$
\eta:\left(\overline{\mathrm{B}_{\rho_{1}}(C)} \cap\left\{u \in X: c_{0}-\delta \leq f(u)<c_{1}+\delta\right\}\right) \times[0,1] \longrightarrow\left\{u \in X: f(u) \geq c_{0}-\delta\right\}
$$

such that

(a) $\tau(u)=0 \Leftrightarrow f(u)=c_{0}-\delta$;

(b) $\|\eta(u, t)-u\| \leq \tau(u) t$;

(c) $f(\eta(u, t)) \leq f(u)-\sigma \tau(u) t$;

(d) $f(\eta(u, 1))=c_{0}-\delta$.

Let $\vartheta_{1}: \mathbb{R} \rightarrow[0,1]$ be a continuous function such that

$$
\vartheta_{1}(s)=1 \quad \text { if } s \leq c_{1}, \quad \vartheta_{1}(s)=0 \quad \text { if } s \geq c_{1}+\frac{\delta}{2},
$$

and let $\vartheta_{2}: X \rightarrow[0,1]$ be a continuous function such that

$$
\vartheta_{2}(u)=1 \quad \text { if }\|u\| \geq R, \quad \vartheta_{2}(u)=0 \quad \text { if }\|u\| \leq R-\delta .
$$

Let $\mathscr{H}: X \times[0,1] \rightarrow X$ be the deformation defined by

$$
\mathscr{H}(u, t)= \begin{cases}\eta\left(u, \vartheta_{1}(f(u)) \vartheta_{2}\left(P_{X_{-}} u\right) t\right) & \text { if } u \in \overline{\mathrm{B}_{\rho_{1}}(C)}, c_{0}-\delta \leq f(u) \leq c_{1}+\delta, \\ u & \text { if } f(u) \leq c_{0}-\delta, \\ u & \text { if } f(u) \geq c_{1}+\frac{\delta}{2}, \\ u & \text { if }\left\|P_{X_{-}} u\right\| \leq R-\delta .\end{cases}
$$


12 Existence of nontrivial solutions for semilinear problems

If $u \in X_{-}$, we have that

$$
\left\|P_{X_{-}} \mathscr{H}(u, t)-u\right\| \leq c\|\mathscr{H}(u, t)-u\| \leq c \frac{f(u)-f(\mathscr{H}(u, t))}{\sigma} \leq c \frac{c_{1}-c_{0}+\delta}{\sigma}<R-r .
$$

It follows

$$
\begin{gathered}
\left\|P_{X_{-}} u\right\| \leq r \Longrightarrow \mathscr{H}(u, t)=u, \\
u \in X_{-}, \quad f(\mathcal{H}(u, 1))<c_{0}, \\
\|u\| \geq R
\end{gathered} \Longrightarrow \underset{\left\|P_{X_{-}}(\mathcal{H}(u, t))\right\| \geq r, \quad \forall t \in[0,1] .}{ }
$$

It is clear that $\left(X,\left(X_{-} \backslash \mathrm{B}_{r}(0)\right) \oplus X_{+}\right)$links $X_{+}$homologically in dimension $\operatorname{dim} X_{-}$and that the inclusion map

$$
i:\left(\overline{\mathrm{B}_{R}(0)} \cap X_{-}, \partial \mathrm{B}_{R}(0) \cap X_{-}\right) \longrightarrow\left(X,\left(X_{-} \backslash \mathrm{B}_{r}(0)\right) \oplus X_{+}\right)
$$

induces an isomorphism in homology. Let $m=\operatorname{dim} X_{-}$and

$$
B=\overline{\mathrm{B}_{R}(0)} \cap X_{-}, \quad E=\partial \mathrm{B}_{R}(0) \cap X_{-}, \quad F=\left(X_{-} \backslash \mathrm{B}_{r}(0)\right) \oplus X_{+} .
$$

Consider now the commutative diagram

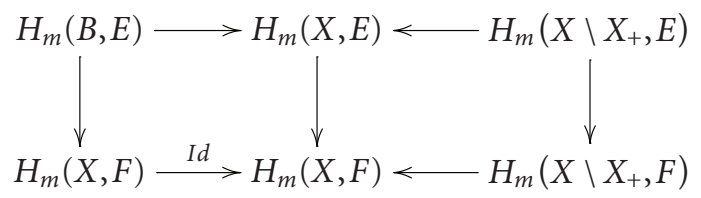

where horizontal rows are induced by the inclusions and the vertical rows are isomorphisms. We have that there exists $z \in H_{m}(X, E)$ belonging to the image of $H_{m}(B, E) \rightarrow$ $H_{m}(X, E)$ such that $i_{*}(z) \in H_{m}(X, F)$, but not to the image of $H_{m}\left(X \backslash X_{+}, F\right) \rightarrow H_{m}(X, F)$. Let us consider the compact sets $D=\mathscr{H}(B, 1)$ and $S=\mathscr{H}(E, 1)$. We have that

$$
\max _{D} f \leq c_{1}, \quad \max _{S} f<c_{0}, \quad S \subseteq F .
$$

Consider now the commutative diagram

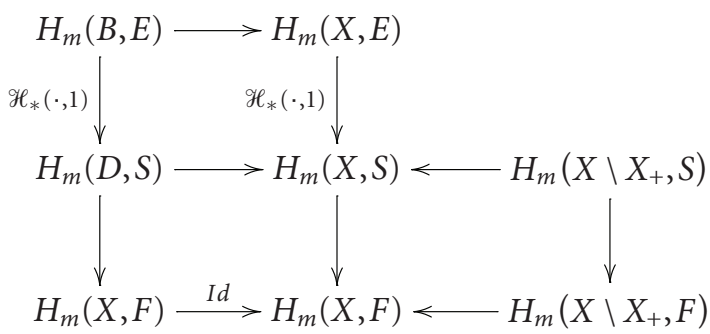


Since $\mathcal{H}(\cdot, 1):(X, E) \rightarrow(X, F)$ is homotopically equivalent to the identity map, then $(D, S)$ links $X_{+}$homologically in dimension $m=\operatorname{dim} X_{-}$and the assertions follows.

\section{Proof of the main results}

Proof of Theorem 2.1. By contradiction, let us assume that 0 is the unique solution of (2.5). Since $m=\operatorname{dim} X_{-} \notin\left[m(f, 0), m^{*}(f, 0)\right]$, by Theorem 3.4 it is $C_{m}(f, 0)=\{0\}$. By Proposition 4.3 there exists a compact pair $(D, S)$ in $H_{0}^{1}(\Omega)$ such that

$$
\forall u \in S: f(u)<\inf _{X_{+}} f
$$

and $(D, S)$ links $X_{+}$homologically in dimension $m$ over all $\mathbb{K}$. By Theorem 4.2 there exists a critical point $u \in H_{0}^{1}(\Omega)$ of $f$ such that $C_{m}(f, u) \neq\{0\}$. Hence $u \neq 0$ and $u$ is a weak solution of (2.5): a contradiction.

proof of Theorem 1.3. Let $(D, S)$ be as in Proposition 4.3. By [13, Proposition 3.9 and Remark] there exists $\delta>0$ such that $f$ satisfies $(P S)_{c}$ for every $c \in\left[c_{0}-\delta, c_{1}+\delta\right]$ and $f^{\prime \prime}(u)$ is a Fredholm operator at every critical point $u$ in $f^{-1}\left(\left[c_{0}-\delta, c_{1}+\delta\right]\right)$. Let us argue by contradiction and set

$$
\begin{gathered}
K_{1}=\left\{u \in H: c_{0}-\delta \leq f(u) \leq c_{1}+\delta, f^{\prime}(u)=0, m^{*}(f, u)<\operatorname{dim} H_{-}\right\}, \\
K_{2}=\left\{u \in H: c_{0}-\delta \leq f(u) \leq c_{1}+\delta, f^{\prime}(u)=0, m(f, u)>\operatorname{dim} H_{-}\right\} .
\end{gathered}
$$

Then $K_{1}, K_{2}$ are two disjoint compact sets whose union is the critical set of $f$ in $f^{-1}\left(\left[c_{0}-\right.\right.$ $\left.\left.\delta, c_{1}+\delta\right]\right)$. By Marino-Prodi perturbation lemma [9, Teorema 2.2], there exists a functional $\hat{f}: H \rightarrow \mathbb{R}$ of class $C^{2}$ such that

$$
\inf _{H_{+}} \hat{f}>c_{0}-\delta / 2, \quad \sup _{H_{-}} \hat{f}<c_{1}+\delta / 2, \quad \max _{S} \hat{f}<\inf _{H_{+}} \hat{f}
$$

$\hat{f}$ satisfies $(P S)_{c}$ for every $c \in\left[c_{0}-\delta / 2, c_{1}+\delta / 2\right], \hat{f}$ has only nondegenerate critical points $u$ in $\hat{f}^{-1}\left(\left[c_{0}-\delta / 2, c_{1}+\delta / 2\right]\right)$, with either $m(\hat{f}, u)<\operatorname{dim} H_{-}$or $m^{*}(\hat{f}, u)>\operatorname{dim} H_{-}$. If we apply Theorem 4.2 to $\hat{f}$, we find a critical point $u$ of $\hat{f}$ with $c_{0}-\delta / 2 \leq \hat{f}(u) \leq c_{1}+\delta / 2$ and $C_{m}(\hat{f}, u) \neq\{0\}$, where $m=\operatorname{dim} H_{-}$. By the Morse lemma, we have $m(\hat{f}, u)=m$ and a contradiction follows.

\section{Acknowledgment}

The author wishes to thank Prof. Marco Degiovanni for helpful discussions and valuable hints. 


\section{Existence of nontrivial solutions for semilinear problems}

\section{References}

[1] H. Amann and E. Zehnder, Nontrivial solutions for a class of nonresonance problems and applications to nonlinear differential equations, Ann. Scuola Norm. Sup. Pisa Cl. Sci. (4) 7 (1980), no. 4, 539-603.

[2] K. C. Chang, Solutions of asymptotically linear operator equations via Morse theory, Comm. Pure Appl. Math. 34 (1981), no. 5, 693-712.

[3] __, Infinite-Dimensional Morse Theory and Multiple Solution Problems, Progress in Nonlinear Differential Equations and Their Applications, vol. 6, Birkhäuser, Massachusetts, 1993.

[4] S. Cingolani and M. Degiovanni, Nontrivial solutions for p-Laplace equations with right-hand side having p-linear growth at infinity, Comm. Partial Differential Equations 30 (2005), no. 8, 1191-1203.

[5] J.-N. Corvellec and A. Hantoute, Homotopical stability of isolated critical points of continuous functionals, Set-Valued Anal. 10 (2002), no. 2-3, 143-164.

[6] A. A. de Moura and F. M. de Souza, A Morse lemma for degenerate critical points with low differentiability, Abstr. Appl. Anal. 5 (2000), no. 2, 113-118.

[7] O. A. Ladyzhenskaya and N. N. Ural'tseva, Linear and Quasilinear Elliptic Equations, Nauka Press, Moscow, 1964, Academic Press, New York, 1968.

[8] A. C. Lazer and S. Solimini, Nontrivial solutions of operator equations and Morse indices of critical points of min-max type, Nonlinear Anal. 12 (1988), no. 8, 761-775.

[9] A. Marino and G. Prodi, Metodi perturbativi nella teoria di Morse, Boll. Un. Mat. Ital. (4) 11 (1975), no. 3, suppl., 1-32.

[10] J. Mawhin and M. Willem, Critical Point Theory and Hamiltonian Systems, Applied Mathematical Sciences, vol. 74, Springer, New York, 1989.

[11] K. Perera and M. Schechter, Morse index estimates in saddle point theorems without a finite dimensional closed loop, Indiana Univ. Math. J. 47 (1998), no. 3, 1083-1095.

[12] Applications of Morse theory to the solution of semilinear problems depending on $C^{1}$ functionals, Nonlinear Anal. Ser. A: Theory Methods 45 (2001), no. 1, 1-9.

[13] S. Solimini, Morse index estimates in min-max theorems, Manuscripta Math. 63 (1989), no. 4, 421-453.

[14] E. H. Spanier, Algebraic Topology, McGraw-Hill, New York, 1966.

Sergio Lancelotti: Dipartimento di Matematica, Politecnico di Torino, Corso Duca degli Abruzzi 24, I 10129 Torino, Italy

E-mail address: sergio.lancelotti@polito.it 


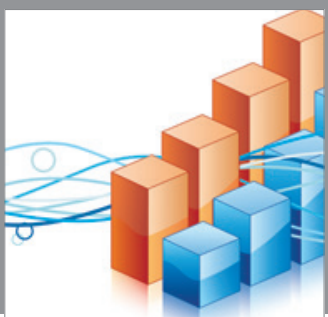

Advances in

Operations Research

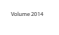

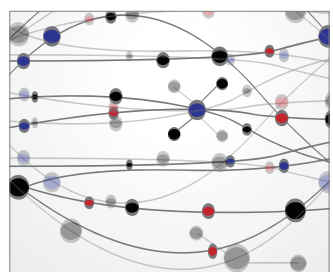

\section{The Scientific} World Journal
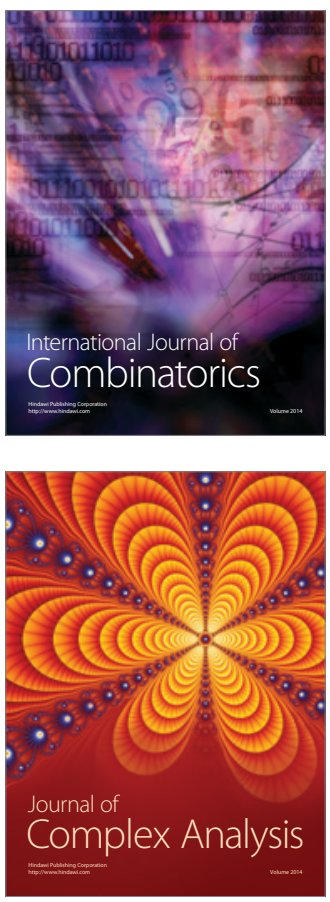

International Journal of

Mathematics and

Mathematical

Sciences
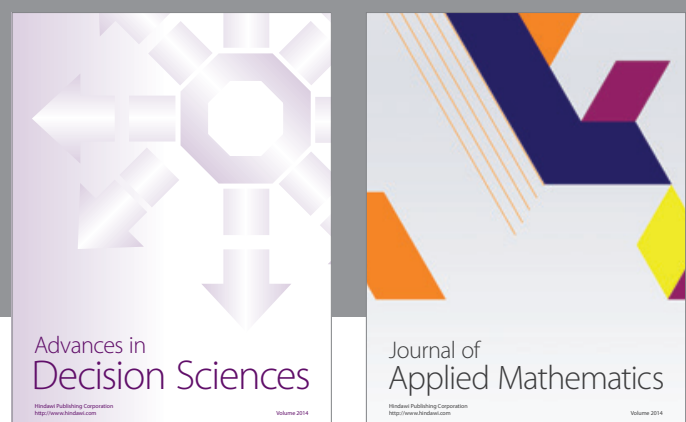

Journal of

Applied Mathematics
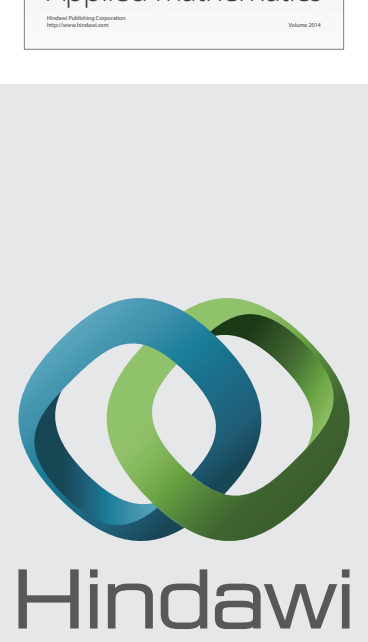

Submit your manuscripts at http://www.hindawi.com
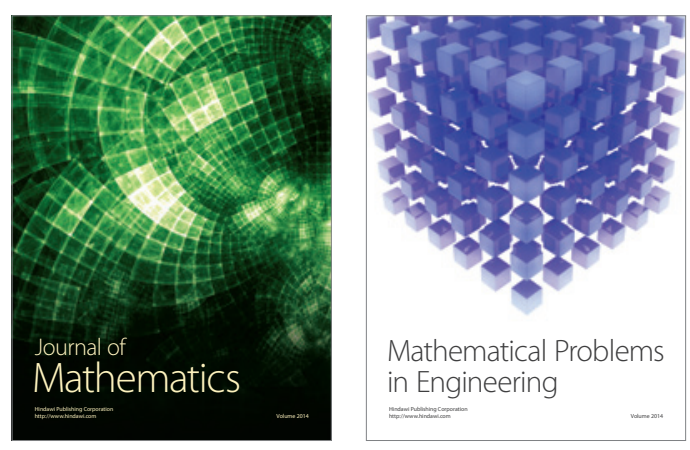

Mathematical Problems in Engineering
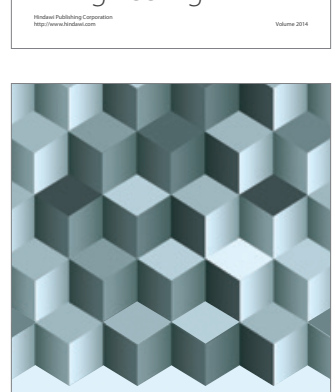

Journal of

Function Spaces
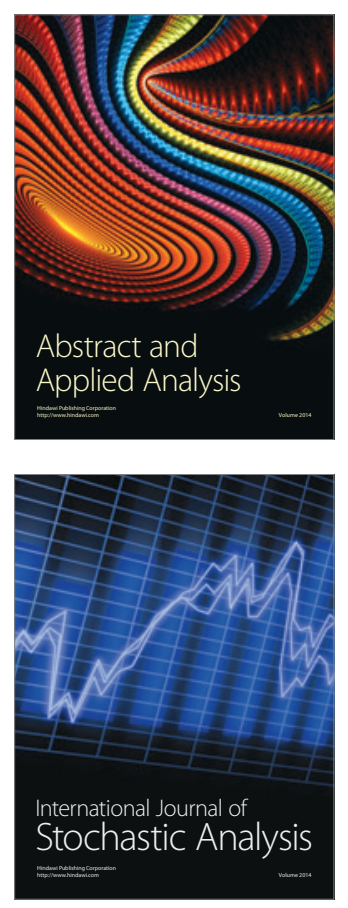

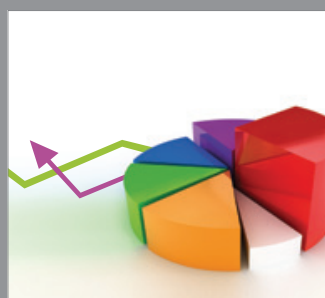

ournal of

Probability and Statistics

Promensencen
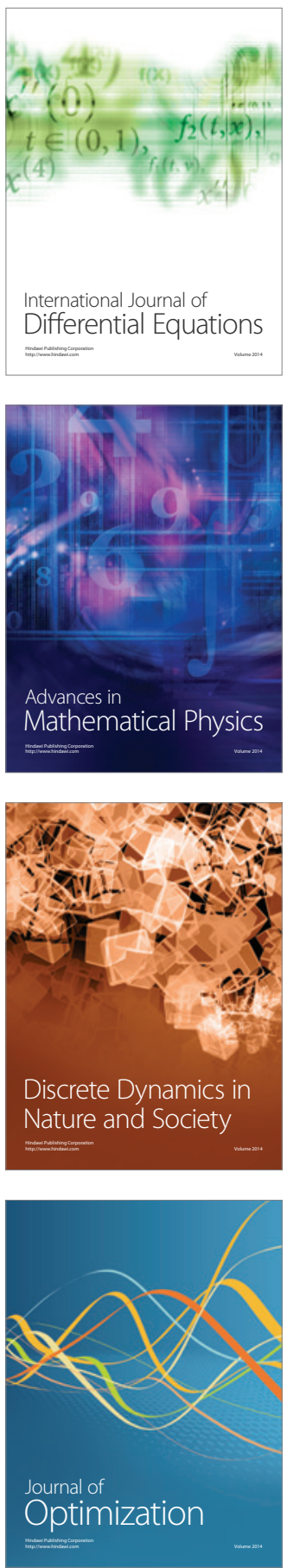